\title{
Habilidade de indicadores antropométricos e de composição corporal em identificar a resistência à insulina
}

\author{
Predictive ability of anthropometric and body composition \\ indicators in the identification of insulin resistance
}

Ana Carolina Junqueira Vasques', Lina Enriqueta Frandsen Paez de Lima Rosado', Gilberto Paixão Rosado', Rita de Cassia Lanes Ribeiro', Sylvia do Carmo Castro Franceschini', Bruno Geloneze ${ }^{2}$, Silvia Eloiza Priore' ${ }^{1}$ Dirce Ribeiro de Oliveira ${ }^{3}$

\section{RESUMO}

Objetivos: Avaliar a habilidade de indicadores antropométricos e de composição corporal em identificar a resistência à insulina (RI), determinando-se os pontos de corte para os que apresentarem melhor eficácia. Métodos: Foram avaliados 138 homens. Determinou-se: perímetro da cintura (PC), diâmetro abdominal sagital (DAS), índice de conicidade (IC), índice de massa corporal (IMC), percentual de gordura corporal (\%GC), índice sagital (IS) e relações cintura-estatura (RCE), cintura-quadril (RCQ) e cintura-coxa (RCCoxa). A RI foi avaliada pelo HOMA-IR. Utilizou-se análise de correlação e análise ROC, com determinação das áreas abaixo da curva (AUC). Resultados: 0 DAS $(r=0,482$; $A \cup C=0,746)$ e o $P C(r=0,464 ; A \cup C=0,739)$ apresentaram correlações mais fortes com o HOMA-IR e maior poder discriminante para $\mathrm{RI}(\mathrm{p}<0,001)$, sendo seus melhores pontos de corte 89,3 cm e 20,0 cm, respectivamente. Conclusão: Os indicadores de obesidade central, o PC e o DAS demonstraram maior habilidade em identificar RI em homens. Encoraja-se a realização de estudos com mulheres e idosos na busca dos melhores pontos de corte para toda a população. Arq Bras Endocrinol Metab. 2009;53(1):72-79.

Descritores

Resistência à insulina; HOMA; perímetro da cintura; diâmetro abdominal sagital; obesidade

Correspondência para: Ana Carolina Junqueira Vasques Departamento de Nutrição e Saúde da UFV, Av. PH Rolfs, s/n,

Campus Universitário

36571-000 Viçosa, MG anacarolinavasques@yahoo.com.br

Recebido em 25/Jul/2008 Aceito em 27/Out/2008

\begin{abstract}
Objective: To assess the ability of anthropometric and body composition indicators in identifying insulin resistance (IR), determining cut-off points for those showing the best efficacy. Method: 138 men were evaluated. Waist perimeter (WP), sagittal abdominal diameter (SAD), conicity index, body mass index (BMI), body fat percent, sagittal index, and the waist-to-height, waist-to-hip and waist-to-thigh ratios were determined. IR was assessed by the HOMA-IR index. Statistical analysis consisted of Spearman correlation coefficient and ROC (receiver operating characteristic) curves, calculating the area under the curve (AUC). Results: SAD ( $r=0.482, A \cup C=0.746)$ and WP ( $r=0.464$, $A \cup C=0.739$ ) showed stronger correlations with the HOMA-IR and greater ability to identify IR $(p<0.001)$, being $89.3 \mathrm{~cm}$ and $20.0 \mathrm{~cm}$ the best cut-offs, respectively. Conclusion: The anthropometric indicators of central obesity, WP and SAD, have shown greater ability to identify IR in men. We encourage studies in women and elderly people in search of the best cut-off points for the entire population. Arq Bras Endocrinol Metab. 2009;53(1):72-79.
\end{abstract}

Keywords

Insulin resistance; HOMA; waist perimeter; sagittal abdominal diameter; obesity 


\section{INTRODUÇÃO}

$\mathrm{I}^{\mathrm{n}}$ divíduos obesos estão mais suscetíveis a desenvolver algum grau de resistência à insulina (RI), que é fator de risco para o desenvolvimento de diabetes tipo 2 e de doença cardiovascular $(1,2)$. Nesse contexto, a avaliação da RI tem recebido considerável atenção, pois a identificação precoce dessa alteração metabólica implica possibilidade de prevenção de doenças, melhora na qualidade de vida e menores gastos em saúde.

Atualmente, o método mais acurado para a determinação da RI é o clampe euglicêmico hiperinsulinêmico. Contudo, é uma técnica dispendiosa, demorada, invasiva e de alta complexidade (3). O índice homeostasis model assessment of insulin resistance (HOMA-IR) representa uma das alternativas para a avaliação da RI, por ser de aplicação mais fácil e por apresentar correlação forte com o clampe (4).

Os indicadores antropométricos e de composição corporal são instrumentos alternativos para a avaliação da RI de maneira acessível, rápida e não-invasiva. Tais indicadores podem ser analisados segundo o tipo de obesidade que eles avaliam: obesidade central, que representa o acúmulo de gordura na região abdominal; obesidade generalizada, que inclui o acúmulo de gordura na periferia e na região central do corpo; e distribuição de gordura corporal. Os indicadores de obesidade central que vêm sendo associados à RI são o diâmetro abdominal sagital (DAS) (5), o perímetro da cintura (PC) (6), o índice de conicidade (ICO) (7) e a relação cintura-estatura (RCE) (8). Para a obesidade generalizada, frequentemente são utilizados o índice de massa corporal (IMC) (9) e o percentual de gordura corporal (\%GC) (10); enquanto para a avaliação da distribuição de gordura corporal tem sido utilizados a relação cintura-quadril (RCQ) (11), a relação cintura-coxa (RCCoxa) (12) e o índice sagital (IS) (13).

Diante da variedade de indicadores de obesidade relacionados à RI, objetivou-se: 1) avaliar o comportamento desses diversos indicadores em relação aos níveis de HOMA-IR em homens adultos; 2) determinar quais desses indicadores são mais eficazes em identificar RI; e 3 ) analisar os pontos de corte para os indicadores que apresentarem melhor eficácia em discriminar os níveis mais elevados do índice HOMA-IR.

\section{MÉTODOS}

Foi realizado um estudo transversal, no qual foram avaliados homens adultos vinculados à Universidade Federal de Viçosa (UFV). Os dados foram coletados na Divisão de Saúde da UFV, localizada em Viçosa, Minas Gerais,
Brasil. O protocolo do estudo foi aprovado pelo Comitê de Ética em Pesquisa com Seres Humanos da UFV.

Os critérios de exclusão foram: glicemia de jejum > 99 $\mathrm{mg} / \mathrm{dL}$, níveis de LDL-colesterol $\geq 160 \mathrm{mg} / \mathrm{dL}$, triglicérides $\geq 150 \mathrm{mg} / \mathrm{dL}$, história prévia de evento cardiovascular, presença de hipertensão arterial e uso de medicamentos com efeitos no metabolismo dos carboidratos e dos lipídeos.

A pressão arterial foi aferida com esfigmomanômetro de mercúrio, sendo considerada normal a pressão arterial sistólica < $140 \mathrm{mmHg}$ e a diastólica $<90 \mathrm{mmHg}$, seguindo as normas da Sociedade Brasileira de Cardiologia (14). Os voluntários que apresentaram valores pressóricos alterados tiveram sua pressão arterial aferida em outros dois momentos distintos. Nas situações em que a pressão arterial permaneceu elevada nas aferições posteriores, os voluntários passaram pela avaliação do médico cardiologista da equipe, a fim de diagnosticar a condição de normotenso ou hipertenso.

\section{AVALIAÇÕES ANTROPOMÉTRICA E DA COMPOSIÇÃO CORPORAL}

As avaliações antropométrica e da composição corporal foram realizadas por um único avaliador treinado. Foram aferidos peso e estatura consoante às técnicas propostas por Jelliffe (15). O PC foi aferido com fita métrica no ponto médio entre a crista ilíaca e a última costela (16). O DAS foi aferido com caliper abdominal (Holtain Kahn Abdominal Caliper $\left.^{\circledR}\right)$ de haste móvel. $\mathrm{O}$ avaliado permaneceu na posição supina, com os joelhos flexionados, sendo a aferição realizada ao nível da menor cintura entre o tórax e o quadril (17). O DAS também foi aferido no ponto médio entre as cristas ilíacas, para o cálculo do IS, segundo referenciado na literatura (18). O perímetro do quadril foi medido na parte mais saliente entre a cintura e a coxa (16). O perímetro da coxa foi aferido do lado direito do corpo, no ponto médio entre a dobra inguinal e a borda proximal da patela. $\mathrm{O}$ avaliado permaneceu em pé e com a perna direita ligeiramente flexionada (19). Todos os perímetros foram tomados em duplicatas e foram calculadas suas respectivas médias. Quando houve diferença $>1 \mathrm{~cm}$ entre as duas medidas, foi realizada terceira medida e utilizados os dois valores mais próximos. Os índices estudados encontram-se na Tabela 1. Para o IMC considerou-se como excesso de peso corporal valores $\geq 25 \mathrm{~kg} / \mathrm{m}^{2}(16)$.

$\mathrm{O}$ \%GC foi avaliado pelo método de bioimpedância tetrapolar amplamente validado na literatura $(20,21)$. Utilizou-se o analisador de bioimpedância horizontal - modelo BIA 450. Antes da realização da medida, cada voluntário foi orientado a seguir o protocolo proposto por Lukaski e cols. (21). Considerou-se como elevado o \%GC $\geq 25 \%$ (22). 


\begin{tabular}{|c|c|c|}
\hline Indicadores & Fórmulas & Referências \\
\hline Índice de conicidade & $\mathrm{PC}(\mathrm{m}) / 0,109 \times \sqrt{\text { peso corporal }(\mathrm{kg})} /$ Estatura $(\mathrm{cm})$ & (7) \\
\hline Relação cintura/estatura & $\mathrm{PC}(\mathrm{cm}) /$ Estatura $(\mathrm{cm})$ & (8) \\
\hline Relação cintura/quadril & $\mathrm{PC}(\mathrm{cm}) / \mathrm{PQ}(\mathrm{cm})$ & (11) \\
\hline Relação cintura/coxa & $\mathrm{PC}(\mathrm{cm}) / \mathrm{PCoxa}(\mathrm{cm})$ & (12) \\
\hline Índice sagital & DAS $(\mathrm{cm}) / \mathrm{PCoxa}(\mathrm{cm})$ & (13) \\
\hline Índice de massa corporal & Peso $(\mathrm{kg}) /$ Estatura $^{2}(\mathrm{~cm})$ & (15) \\
\hline
\end{tabular}

$\mathrm{PC}=$ perímetro da cintura; $\mathrm{PQ}$ = perímetro do quadril; $\mathrm{PCoxa}=$ perímetro da coxa; $\mathrm{DAS}$ = diâmetro abdominal sagital.

\section{ANÁLISES BIOQUÍMICAS}

Foram determinados: glicose e insulina plasmáticas de jejum, colesterol total, HDL, LDL e triglicérides. As amostras de sangue foram coletadas após jejum noturno de 12 horas. As determinações plasmáticas dos triglicérides, colesterol total, HDL-colesterol e glicose foram realizadas pelo método colorimétrico enzimático. A fração LDL-colesterol foi determinada segundo a fórmula de Friedwald (23). A insulina plasmática foi determinada pelo método Elisa, com a utilização de kits ultrassensíveis e coeficientes de variação intraensaio e interensaio de $5,96 \pm 1,17 \mu \mathrm{U} / \mathrm{mL}$ e $10,3 \pm 0,9 \mu \mathrm{U} / \mathrm{mL}$, respectivamente. $\mathrm{O}$ método utilizado para a avaliação da RI foi o índice HOMA-IR calculado a partir da fórmula (24):

$$
\text { HOMA-IR }=\mathrm{IJ}(\mu \mathrm{U} / \mathrm{mL} \times \mathrm{GJ}(\mathrm{mmol} / \mathrm{L}) / 22,5
$$

em que IJ corresponde à insulinemia de jejum e GJ à glicemia de jejum. Considerou-se como ponto de corte para as análises o valor referente ao percentil 75 do HOMA-IR na amostra avaliada.

\section{ANÁLISES ESTATÍSTICAS}

As análises estatísticas foram realizadas nos programas SPSS 12.0 e MedCalc 9.3. O nível de significância adotado foi inferior a $5 \%(\mathrm{p}<0,05)$. Para as variáveis com distribuição normal, utilizou-se a análise de variância com o teste post-hoc de Tukey e o cálculo do coeficiente de correlação de Pearson. Para as variáveis que não apresentaram distribuição normal, empregou-se o teste de Kruskall-Wallis com o teste post-hoc de Dunn's e o cálculo do coeficiente de correlação de Spearman. Foram construídas curvas ROC (receiver operating characteristic curve) e calculadas as áreas abaixo das curvas, com intervalo de confiança de 95\% (25). O teste $\mathrm{Z}$ foi utilizado para comparação das áreas abaixo das curvas. Os valores de sensibilidade (S) e especificidade (E) dos indicadores antropométricos foram calculados para cada ponto de corte presente na amostra. O ponto de corte com maior soma entre $S$ e $E$ foi escolhido por otimizar a relação entre estes dois parâmetros, atentando-se para que os valores mínimos de $\mathrm{S}$ e $\mathrm{E}$ fossem $\geq 60 \%$ (26).

\section{RESULTADOS}

A amostra do presente estudo foi constituída por 138 homens adultos com idade entre 20 a 59 anos. Na Tabela 2 , estão apresentados os dados clínicos e laboratoriais dos indivíduos avaliados. Quanto ao estado nutricional, segundo valores de IMC, 44,9\% apresentaram excesso de peso corporal e $31,2 \%$ estavam com o \%GC elevado. Na Tabela 3, é possível observar que a distribuição dos indicadores antropométricos e de composição corporal apresentou diferenças estatísticas significantes com o aumento progressivo para todos os 
indicadores aumento do HOMA-IR. Ressalta-se que para o IMC e para os indicadores de obesidade central, com exceção do ICO, as diferenças entre os quartis foram ainda mais acentuadas.

\begin{tabular}{|c|c|c|}
\hline Variáveis & Média \pm DP & Mediana (min.-máx.) \\
\hline Idade (anos) & $38,4 \pm 10,7$ & $39,0(20-59)$ \\
\hline Peso (kg) & $73,3 \pm 10,2$ & $73,8(45,7-98,0)$ \\
\hline Estatura (cm) & $173,4 \pm 6,9$ & $174,2(154,5-194,4)$ \\
\hline IMC (kg/m²) & $24,4 \pm 3,1$ & $24,6(17,08-34,7)$ \\
\hline $\mathrm{PC}(\mathrm{cm})$ & $87,2 \pm 9,3$ & $87,8(68,2-110,3)$ \\
\hline DAS (cm) & $19,4 \pm 2,3$ & $19,6(14,1-26,2)$ \\
\hline $\mathrm{RCQ}$ & $0,87 \pm 0,1$ & $0,87(0,76-1,05)$ \\
\hline RCCoxa & $1,57 \pm 0,2$ & $1,56(0,46-1,90)$ \\
\hline RCE & $0,50 \pm 0,1$ & $0,50(0,38-0,68)$ \\
\hline ICO & $1,23 \pm 0,1$ & $1,23(1,08-1,44)$ \\
\hline IS & $0,35 \pm 0,5$ & $0,35(0,29-0,45)$ \\
\hline$\% G C(\%)$ & $21,6 \pm 5,4$ & $21,5(6,4-32,9)$ \\
\hline Glicemia de jejum (mg/dL) & $83,3 \pm 7,0$ & $83,0(60,0-99,0)$ \\
\hline HOMA-IR & $1,13 \pm 0,35$ & $1,06(0,52-2,43)$ \\
\hline Colesterol total (mg/dL) & $162,61 \pm 29,43$ & $163,0(84,0-220,0)$ \\
\hline HDL-Colesterol (mg/dL) & $44,9 \pm 12,6$ & $43,5(23,0-110,0)$ \\
\hline LDL-Colesterol (mg/dL) & $102,22 \pm 26,88$ & $103,3(31,2-156,2)$ \\
\hline Triglicérides (mg/dL) & $77,21 \pm 28,72$ & $74,50(24,0-146,0)$ \\
\hline
\end{tabular}

$\mathrm{DP}=$ desvio-padrão; $\mathrm{IMC}=$ índice de massa corporal; $\mathrm{PC}=$ perímetro da cintura; $\mathrm{DAS}=$ diâmetro abdominal sagital; $\mathrm{RCQ}=$ relação cintura-quadril; $\mathrm{RCCoxa}=$ relação cintura-coxa; RCE = relação cintura-estatura; ICO = índice de conicidade; IS = índice sagital; \%GC $=\%$ gordura corporal.
Os resultados das análises de correlação entre os indicadores de obesidade e o HOMA-IR encontram-se na Tabela 4. De modo geral, os indicadores de obesidade central, representados pelo DAS seguido do PC, apresentaram as melhores correlações com o HOMA-IR.

\begin{tabular}{lc}
\hline $\begin{array}{l}\text { Tabela 4. Correlações entre os indicadores antropométricos e } \\
\text { de composição corporal e o HOMA-IR. }\end{array}$ \\
\hline Variáveis & HOMA-IR \\
\hline Obesidade central & $0,464^{\ddagger}$ \\
PC & $0,482^{\ddagger}$ \\
DAS & $0,370^{\ddagger}$ \\
ICO & $0,406^{\ddagger}$ \\
RCE & \\
Obesidade generalizada & $0,377^{\ddagger}$ \\
IMC (kg/m²) & $0,394^{\ddagger}$ \\
\%GC & \\
Distribuição de gordura corporal & $0,379^{\ddagger}$ \\
RCQ & $0,345^{\ddagger}$ \\
RCCoxa & $0,350^{\ddagger}$ \\
IS
\end{tabular}

Coeficiente de correlação de Spearman: $\ddagger p<0,001 ; P C=$ perímetro da cintura; DAS = diâmetro abdominal sagital; ICO = índice de conicidade; RCE = relação cinturaestatura; IMC = índice de massa corporal; \%GC = \% gordura corporal; RCQ = relação cintura-quadril; RCCoxa = relação cintura-coxa; IS = índice sagital.

Na análise ROC (Figura 1), o PC e o DAS apresentaram, simultaneamente, os maiores valores absolutos para as áreas abaixo da curva, os intervalos de confiança mais elevados e os menores erros-padrão (Tabela 5). A aplicação do teste Z para comparações das áreas abaixo da curva evidenciou que

Tabela 3. Distribuição dos indicadores antropométricos e de composição corporal segundo quartis de HOMA-IR nos indivíduos avaliados.

\begin{tabular}{|c|c|c|c|c|}
\hline Variáveis & $\begin{array}{c}1^{0} \text { quartil } \\
\text { HOMA } \\
\leq 0,90 \\
n=35\end{array}$ & $\begin{array}{c}2^{0} \text { quartil } \\
\text { HOMA } \\
0,91-1,06 \\
n=35\end{array}$ & $\begin{array}{c}3^{0} \text { quartil } \\
\text { HOMA } \\
1,07-1,28 \\
n=34\end{array}$ & $\begin{array}{c}4^{0} \text { quartil } \\
\text { HOMA } \\
\geq 1,29 \\
n=34\end{array}$ \\
\hline \multicolumn{5}{|c|}{ Obesidade central } \\
\hline PC (cm)‡ & $81,5 \pm 7,5 \mathrm{a}$ & $86,1 \pm 9,6 a b$ & $88,8 \pm 7,4 b c$ & $92,6 \pm 8,9 c$ \\
\hline DAS (cm)‡ & $17,9 \pm 1,8 \mathrm{a}$ & $19,0 \pm 2,4 a b$ & $19,9 \pm 1,9 b c$ & $20,8 \pm 2,2 \mathrm{C}$ \\
\hline ICO‡ & $1,19 \pm 0,06 a$ & $1,23 \pm 0,07 a b$ & $1,23 \pm 0,07 a b$ & $1,26 \pm 0,07 b$ \\
\hline RCE‡ & $0,47 \pm 0,04 a$ & $0,50 \pm 0,06 a b$ & $0,51 \pm 0,04 b c$ & $0,53 \pm 0,06 \mathrm{c}$ \\
\hline \multicolumn{5}{|c|}{ Obesidade generalizada } \\
\hline IMC (kg/m²)‡ & $22,9 \pm 2,7 \mathrm{a}$ & $23,6 \pm 3,2 a b$ & $25,0 \pm 2,5 b c$ & $25,9 \pm 3,0 \mathrm{c}$ \\
\hline$\% \mathrm{GC}(\%) \ddagger$ & $18,5 \pm 4,6 \mathrm{a}$ & $21,3 \pm 5,7 a b$ & $22,8 \pm 4,4 b$ & $22,9 \pm 5,1 b$ \\
\hline \multicolumn{5}{|c|}{ Distribuição de gordura corporal } \\
\hline RCQ $\ddagger$ & $0,85 \pm 0,05 a$ & $0,88 \pm 0,07 a b$ & $0-, 88 \pm 0,05 a b$ & $0,90 \pm 0,06 b$ \\
\hline RCCoxat & $1,51(0,46-1,71) a$ & $1,56(1,40-1,88) b$ & $1,60(1,35-1,90) a b$ & $1,64(1,36-1,89) b$ \\
\hline ISł & $0,33 \pm 0,02 \mathrm{a}$ & $0,35 \pm 0,02 b$ & $0,35 \pm 0,03 \mathrm{~b}$ & $0,36 \pm 0,03 \mathrm{~b}$ \\
\hline
\end{tabular}

ANOVA e teste post hoc de Tuckey para as variáveis apresentadas em média \pm desvio-padrão e Kruskall-Wallis e teste post hoc de Dunn's para as variáveis apresentadas em mediana (valores mínimo e máximo): $† p<0,01 ; \ddagger p<0,001$. Comparações realizadas entre colunas: letras iguais indicam valores iguais e letras diferentes indicam valores com diferença estatística. PC = perímetro da cintura; DAS = diâmetro abdominal sagital; ICO = índice de conicidade; RCE = relação cintura-estatura; IMC = índice de massa corporal; \%GC = \% gordura corporal; RCQ = relação cintura-quadril; RCCoxa = relação cintura-coxa; IS = índice sagital. 


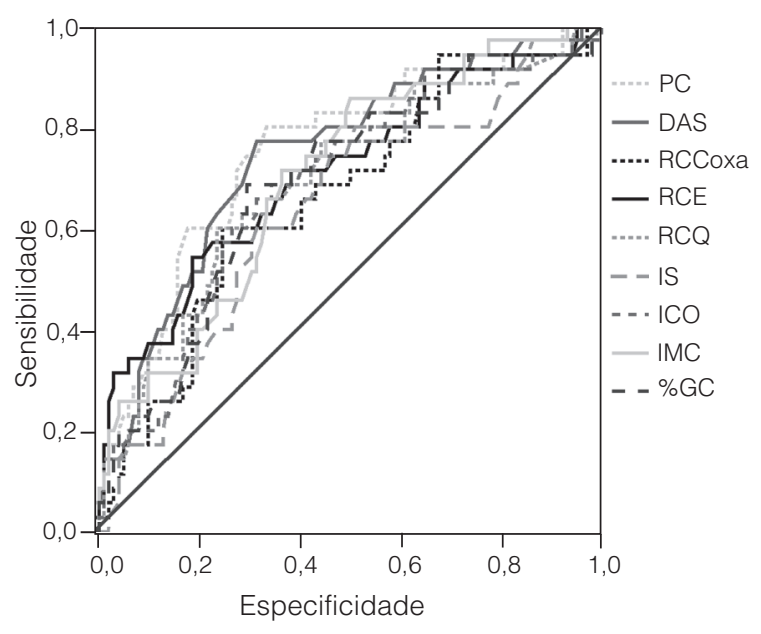

$\mathrm{PC}=$ perímetro da cintura; DAS = diâmetro abdominal sagital; $\mathrm{RCCoxa}=$ relação cintura-coxa; $R C E$ = relação cintura-estatura; $R C Q$ = relação cintura-quadril; $I S=$ índice sagital; ICO = índice de conicidade; IMC = índice de massa corporal; \%GC = \% gordura corporal.

Figura 1. Curva ROC comparando os diferentes indicadores antropométricos e de composição corporal na avaliação do índice HOMA-IR.

o PC e o DAS apresentaram maior área em relação ao IS $(\mathrm{p}<0,05)$. Para o PC, notou-se tendência estatística de que sua área poderia ser maior que as áreas encontradas para a $\operatorname{RCE}(\mathrm{p}=0,064)$, para o ICO $(\mathrm{p}=0,064)$ e para a RCCoxa $(\mathrm{p}=0,063)$. As áreas abaixo da curva para os demais indicadores não diferiram entre si do ponto de vista estatístico. $\mathrm{Na}$ avaliação dos pontos de corte com maior acurácia para cada indicador, o PC e o DAS alcançaram a maior soma entre os valores de S e E para os pontos de corte de 89,3 e 20,0 cm, respectivamente. Estes dois indicadores de obesidade apresentaram melhor eficácia em identificar RI com menor quantidade de falsos-positivo e falsos-negativo (Tabela 5).

\section{DISCUSSÃO}

Os indicadores de obesidade central, em particular o DAS e o PC, se sobressaíram perante aos demais, embora todas as correlações tenham sido de magnitude moderada. Na análise ROC, o mesmo comportamento foi encontrado para estes dois indicadores, visto que o PC apresentou o melhor desempenho. Dois trabalhos $(5,6)$ encontraram melhor eficácia para o DAS e para o PC, respectivamente, em identificar $\mathrm{RI}$ em homens, comparados ao IMC e à RCQ. Em estudo com homens nãoobesos, foi identificada diferença na força das correlações entre os indicadores antropométricos e o nível de RI. Nesse estudo, o PC e o DAS foram as principais variáveis associadas à RI, comparadas à RCQ, ao ICO e ao \%GC (27). Em outro trabalho, o PC e o DAS foram os indicadores antropométricos que melhor se correlacionaram com a insulinemia de jejum comparados à RCQ (28).

O PC e o DAS são medidas que avaliam diretamente a extensão da obesidade abdominal e apresentam correlação forte com a quantidade de gordura visceral (18), que está diretamente relacionada às alterações metabólicas típicas da síndrome metabólica, como a RI. Para o DAS, o fato de

\begin{tabular}{|c|c|c|c|c|c|}
\hline Variáveis & $\begin{array}{r}\text { Área } \pm \text { EP } \\
\text { (IC } 95 \%)\end{array}$ & $\begin{array}{c}\text { Ponto } \\
\text { de corte }\end{array}$ & $\begin{array}{l}\text { Sensibilidade } \\
\text { (IC 95\%) }\end{array}$ & $\begin{array}{l}\text { Especificidade } \\
\text { (IC 95\%) }\end{array}$ & $S+E$ \\
\hline $\mathrm{PC}(\mathrm{cm})$ & $0,746 \pm 0,049^{\ddagger}(0,649-0,842)$ & 89,3 & $\begin{array}{c}80,0 \\
(63,1-91,5)\end{array}$ & $\begin{array}{c}66,0 \\
(56,0-75,1)\end{array}$ & 146,0 \\
\hline DAS (cm) & $0,739 \pm 0,049^{\ddagger}(0,643-0,834)$ & 20,0 & $\begin{array}{c}77,1 \\
(59,9-89,5)\end{array}$ & $\begin{array}{c}68,0 \\
(58,0-76,8)\end{array}$ & 145,1 \\
\hline ICO & $0,682 \pm 0,052^{\dagger}(0,579-0,784)$ & 1,25 & $\begin{array}{c}65,7 \\
(47,8-80,9)\end{array}$ & $\begin{array}{c}67,9 \\
(58,0-76,8)\end{array}$ & 133,6 \\
\hline RCE & $0,702 \pm 0,054^{\ddagger}(0,597-0,807)$ & 0,51 & $\begin{array}{c}62,9 \\
(44,9-78,5)\end{array}$ & $\begin{array}{c}65,1 \\
(55,0-74,2)\end{array}$ & 128,0 \\
\hline IMC (kg/m²) & $0,696 \pm 0,049^{\dagger}(0,599-0,793)$ & 24,8 & $\begin{array}{c}71,4 \\
(53,7-85,3)\end{array}$ & $\begin{array}{c}63,1 \\
(53,0-72,4)\end{array}$ & 134,5 \\
\hline$\% \mathrm{GC}(\%)$ & $0,684 \pm 0,052^{\dagger}(0,582-0,786)$ & 23,1 & $\begin{array}{c}68,6 \\
(50,7-83,1)\end{array}$ & $\begin{array}{c}69,9 \\
(60,1-78,5)\end{array}$ & 138,5 \\
\hline $\mathrm{RCQ}$ & $0,687 \pm 0,053^{\dagger}(0,583-0,791)$ & 0,90 & $\begin{array}{c}60,0 \\
(44,9-78,5)\end{array}$ & $\begin{array}{c}75,7 \\
(61,1-79,4)\end{array}$ & 135,7 \\
\hline RCCoxa & $0,659 \pm 0,053^{\dagger}(0,555-0,762)$ & 1,63 & $\begin{array}{c}60,0 \\
(42,1-76,1)\end{array}$ & $\begin{array}{c}74,8 \\
(65,2-82,8)\end{array}$ & 134,8 \\
\hline IS & $0,636 \pm 0,054^{\S}(0,529-0,742)$ & 0,35 & $\begin{array}{c}62,9 \\
(42,1-76,1)\end{array}$ & $\begin{array}{c}65,1 \\
(54,0-73,3) \\
\end{array}$ & 128,0 \\
\hline
\end{tabular}

${ }^{\S} p<0,05 ;{ }^{\dagger} p<0,01 ;{ }^{\ddagger} p<0,001 ; E P$ = erro-padrão; IC = intervalo de confiança; $S=$ sensibilidade; $E$ = especificidade; $P C$ = perímetro da cintura; $D A S=$ diâmetro abdominal sagital; ICO = índice de conicidade; RCE = relação cintura-estatura; IMC = índice de massa corporal; \%GC = \% gordura corporal; $R C Q=$ relação cintura-quadril; RCCoxa = relação cinturacoxa; IS = índice sagital. 
a medida ter sido aferida com o avaliado na posição supina apresenta vantagens. Nessa posição, o tecido adiposo visceral tende a elevar a parede abdominal na direção sagital e o tecido adiposo abdominal subcutâneo anterior ou lateral comprime o abdômen ou tende a descer para os lados por causa da força da gravidade. Assim, espera-se que o DAS aferido na posição supina reflita, principalmente, o tecido adiposo visceral (29). Ressaltam-se as vantagens técnicas das medidas do PC e do DAS, como a rapidez na execução e a não-utilização de fórmulas. O DAS conta com a desvantagem de necessitar da posse de caliper abdominal e mesa de superfície firme, ao contrário do $\mathrm{PC}$, que requer apenas fita métrica apropriada.

Embora trabalhos realizados com populações diferentes tenham encontrado resultados positivos na identificação de RI para os demais indicadores de obesidade aqui estudados $(8,9,12,13,18)$, nenhum índice mostrou-se superior às medidas do PC e do DAS. Além do mais, desconhece-se a presença de trabalhos realizados nesta população comparando todos estes indicadores de obesidade na identificação da RI, simultaneamente. Acredita-se que o uso de índices antropométricos é pertinente quando sua aplicação apresenta vantagens em relação ao uso da medida isolada, o que não ocorreu com os resultados do presente estudo.

Quanto aos indicadores de obesidade generalizada, IMC e \%GC, seus piores desempenhos no presente estudo no que diz respeito ao PC e ao DAS podem ser explicados pela associação entre gordura visceral e aumento na RI de maneira independente da adiposidade corporal total, segundo a literatura (30). Para a RCE e para o ICO, as vantagens em sua aplicação estariam relacionadas ao ajuste para estatura presente em suas fórmulas, possibilitando, supostamente, comparações diretas entre diferentes populações $(7,8)$. Contudo, para o ICO, sua aplicação na prática pode se tornar dificultada pela equação presente em seu denominador. A RCQ, embora seja o índice de distribuição de gordura corporal mais conhecido e utilizado, é parcialmente independente da adiposidade total, uma vez que indivíduos magros e obesos podem apresentar o mesmo valor de RCQ, dificultando sua interpretação (28). A RCCoxa e o IS foram propostos como alternativas à RCQ para a estimativa da distribuição de gordura corporal e para a predição de morbidades por utilizarem o perímetro da coxa, que não é afetado por variações na arquitetura pélvica, como é o perímetro do quadril (31). Contudo, nas análises de correlação e nas curvas ROC, a RCQ apresentou maiores valores que a RCCoxa e o IS.

$\mathrm{Na}$ prática, um instrumento para screening populacional ou para avaliação do risco de morbidade nos serviços de saúde deve ser sensível, o que resulta na detecção do maior número possível de verdadeiros-positivo, mesmo à custa de maior número de falsos-positivo pela perda de especificidade. Todavia, certo equilíbrio entre os dois parâmetros também deve ser alcançado, uma vez que a especificidade auxilia na racionalização de custos em saúde (26). No presente estudo, além do PC e do DAS terem apresentado a melhor eficácia para avaliação do risco de RI, estes indicadores de obesidade também apresentaram os pontos de corte com a melhor combinação entre sensibilidade e especificidade, pois alcançaram maior acurácia (maior quantidade de verdadeiros-negativo e verdadeiros-positivo), maior sensibilidade e especificidade aceitável.

Em 1995, estudo conduzido com homens europeus demonstrou que valores de PC acima de 94 e $102 \mathrm{~cm}$ indicavam risco aumentado, ou muito aumentado, respectivamente, de complicações metabólicas (32). Mais recentemente, o NCEP-ATP III (33) adotou o valor de $102 \mathrm{~cm}$ para o diagnóstico de obesidade central em homens, o qual tem sido utilizado inclusive no Brasil (34).

As populações diferem entre si de acordo com o nível de risco apresentado para dado PC. Assim, a determinação de pontos de corte para o PC globalmente aplicáveis é impossível (16). A International Diabetes Federation (IDF) (35) recomenda, que para populações originárias das Américas Central e do Sul, seja adotado o mesmo ponto de corte sugerido para a população asiática $(90 \mathrm{~cm}$ para os homens) até que estudos mais específicos com esta população sejam realizados.

No presente trabalho, o valor de PC de $89,3 \mathrm{~cm}(\mathrm{~S}=80 \%$ e $\mathrm{E}=66 \%$ ), identificado como o melhor ponto de corte para a determinação do risco de RI, mostrou-se bastante próximo ao ponto de corte sugerido pela IDF para a utilização do PC como um dos critérios para o diagnóstico de síndrome metabólica. $\mathrm{Na}$ amostra em questão, o valor de $90 \mathrm{~cm}$ sugerido pela IDF apresentou sensibilidade de $74,3 \%$ e especificidade de 70\% (dados não apresentados). Dois estudos realizados no Brasil identificaram pontos de corte para o PC próximos ao encontrado no presente estudo. Vale ressaltar que nesses trabalhos o PC foi aferido no ponto médio entre a crista ilíaca e a última costela, o que favorece as comparações. $\mathrm{O}$ primeiro deles (19) identificou que o melhor ponto de corte do PC para a predição de quantidades aumentadas de tecido adiposo visceral é o valor de $88,7 \mathrm{~cm}(\mathrm{~S}=83 \%$ e $\mathrm{E}=71 \%)$ para homens adultos. O segundo trabalho (26) avaliou ampla amostra $(\mathrm{n}=1.439)$ e sugeriu valores de PC acima de $88 \mathrm{~cm}(\mathrm{~S}=68,7 \%$ e $\mathrm{E}=67,7 \%)$ para diagnóstico da obesidade central nos homens brasileiros.

De acordo com os resultados do presente trabalho e dos estudos discutidos anteriormente realizados com a população brasileira, parece que os pontos de corte pro- 
postos pelo NCEP-ATP III, adotados por entidades em saúde e publicados em diretrizes, podem não ser os mais apropriados para as características da população brasileira. $\mathrm{Na}$ amostra do presente estudo, o ponto de corte de 102 $\mathrm{cm}$ apresentou sensibilidade muito baixa de $11,4 \%$ e especificidade de $71 \%$. Abaixo do valor de $102 \mathrm{~cm}$ a população do presente estudo pode estar em risco de apresentar alterações metabólicas, como RI e dislipidemias, deixando muitas pessoas de fora das medidas de prevenção para o diabetes tipo 2 e as doenças cardiovasculares.

Para o DAS, não existem pontos de corte estabelecidos para sua classificação, provavelmente por ser menos conhecido, mas com grande potencial para começar a ser utilizado. A mesma linha de raciocínio referente ao componente étnico também deve ser aplicada para este indicador. No presente estudo, o melhor ponto de corte identificado para avaliação do risco de RI foi o valor de $20 \mathrm{~cm}$. O trabalho de Sampaio e cols. (19), realizado com homens brasileiros, sugeriu o ponto de corte de $20,5 \mathrm{~cm}(\mathrm{~S}=83 \%$ e $\mathrm{E}=82 \%)$ para o DAS, bastante próximo ao identificado no estudo em questão. Nesse trabalho, o ponto de corte sugerido foi fundamentado em uma área de gordura abdominal visceral elevada, não sendo avaliada a presença de morbidades ou alterações metabólicas.

Para o IMC, é interessante observar que o melhor ponto de corte identificado nas curvas ROC foi o valor de $24,8 \mathrm{~kg} / \mathrm{m}^{2}$, bem próximo ao valor de $25,0 \mathrm{~kg} / \mathrm{m}^{2}$ considerado como diagnóstico de excesso de peso corporal pela Organização Mundial da Saúde (16). Mesmo não tendo apresentado o melhor desempenho, comparado ao DAS e ao PC, como este índice representa indicador do estado nutricional bastante utilizado na prática clínica, acredita-se que possa ser utilizado, em associação ao PC e ao DAS, como mais um indicador do risco de RI.

Embora os valores de sensibilidade e especificidade tenham sido adequados, é importante observar que, para o PC e para o DAS, seus valores de especificidade resultam taxas de $34 \%$ e $32 \%$ de falsos-positivo, respectivamente. Dessa forma, é importante considerar que ambos fornecem estimativas do risco e não diagnósticos de RI, embora as medidas terapêuticas para melhora na RI envolvam a prática regular de exercício físico, dieta equilibrada e manutenção de peso corporal adequado, as quais não apresentam risco à saúde daqueles com o resultado falso-positivo.

No presente trabalho, foram avaliados indivíduos saudáveis vinculados à universidade federal de referência nacional, resultando amostra formada por indivíduos das mais diversas regiões do país, o que, de certa forma, garantiu boa representatividade do componente étnico da população. Assim, sugere-se a utilização dos pontos de corte, de $89,3 \mathrm{~cm}$ para o PC e de $20 \mathrm{~cm}$ para o DAS, como indicadores de risco para RI em homens brasileiros adultos.
Em conclusão, o PC e o DAS foram os indicadores de obesidade mais promissores para a identificação de RI. Acredita-se ser pertinente a avaliação da eficácia dos demais indicadores de obesidade em outros extratos populacionais, uma vez que todos apresentaram correlação com o HOMA-IR, embora com menor magnitude. Todavia, ainda que seja extremamente útil investigar instrumentos alternativos para a identificação de RI, é importante reconhecer que são indicadores de risco e não de diagnóstico. Variáveis importantes no desenvolvimento de RI, como o estilo de vida e a história familiar, sempre deverão ser considerados nas avaliações. Como a distribuição de gordura corporal é influenciada pela etnia, encoraja-se a realização de estudos específicos com a população brasileira, envolvendo a participação de homens e mulheres de diferentes faixas etárias, na busca dos melhores pontos de corte para o PC e para o DAS na avaliação do risco de RI. Embora a RI não seja considerada morbidade, se não controlada por meio de intervenções precoces, pode resultar doenças com sérias complicações de saúde.

Agradecimentos: Os autores agradecem ao Conselho Nacional de Desenvolvimento Científico e Tecnológico $(\mathrm{CNPq})$ e à Fundação de Amparo à Pesquisa do Estado de Minas Gerais (Fapemig) pelo apoio financeiro e ao Laboratório de Investigação em Metabolismo e Diabetes (Limed) da Universidade Estadual de Campinas (Unicamp) pela parceria científica.

Declaração: Os autores declaram não haver conflitos de interesse científico neste artigo.

\section{REFERÊNCIAS}

1. Despres JP, Lemieux I. Abdominal obesity and metabolic syndrome. Nature. 2006;444(7121):881-7.

2. Reaven G. All obese individuals are not created equal: insulin resistance is the major determinant of cardiovascular disease in overweight/ obese individuals. Diab Vasc Dis Res. 2005;2(3):105-12.

3. Geloneze B, Tambascia MA. Avaliação laboratorial e diagnóstico de resistência insulínica. Arq Bras Endocrinol Metabol. 2006;50(2):208-15.

4. Vasques ACJ, Rosado LEFPL, Alfenas RCG, Geloneze B. Análise crítica do uso dos índices do homeostasis model assessment (HOMA) na avaliação da resistência à insulina e capacidade funcional das células-b pancreáticas. Arq Bras Endocrinol Metabol. 2008;52(1):32-9.

5. Riserus U, Arnlov J, Brismar K, Zethelius B, Berglund L, Vessby B. Sagittal abdominal diameter is a strong anthropometric marker of insulin resistance and hyperproinsulinemia in obese men. Diabetes Care. 2004;27(8):2041-6.

6. Ybarra J, Sanchez-Hernandez J, Pou J, Fernández S, Gich I, OrdóñezLlanos J, et al. Anthropometrical measures are easily obtainable sensitive and specific predictors of insulin resistance in healthy individuals. Prevention Control. 2005;1(2):175-81.

7. Valdez R, Seidell JC, Ahn YI, Weiss KM. A new index of abdominal adiposity as an indicator of risk for cardiovascular disease. A crosspopulation study. Int J Obes Relat Metab Disord. 1993;17(2):77-82.

8. Ho SY, Lam TH, Janus ED. Waist to stature ratio is more strongly associated with cardiovascular risk factors than other simple anthropometric indices. Ann Epidemiol. 2003;13(10):683-91. 
9. Chang SA, Kim HS, Yoon KH, Ko SH, Kwon HS, Kim SR, et al. Body mass index is the most important determining factor for the degree of insulin resistance in non-obese type 2 diabetic patients in Korea. Metab Clin Exp. 2004;53(2):142-6.

10. Shen W, Punyanitya M, Chen J, Gallagher D, Albu J, Pi-Sunyer X, et al. Waist circumference correlates with metabolic syndrome indicators better than percentage fat. Obesity (Silver Spring). 2006;14(4):727-36.

11. Pitanga FJ, Lessa I. Anthropometric indexes of obesity as an instrument of screening for high coronary risk in adults in the city of Salvador-Bahia. Arq Bras Cardiol. 2005;85(1):26-31.

12. Chuang YC, Hsu KH, Hwang CJ, Hu PM, Lin TM, Chiou WK. Waist-tothigh ratio can also be a better indicator associated with type 2 diabetes than traditional anthropometrical measurements in Taiwan population. Ann Epidemiol. 2006;16(5):321-31.

13. Kahn HS, Austin H, Williamson DF, Arensberg D. Simple anthropometric indices associated with ischemic heart disease. J Clin Epidemiol. 1996;49(9):1017-24.

14. Sociedade Brasileira de Cardiologia. IV Diretriz Brasileira sobre Dislipidemias e Prevenção da Aterosclerose. Departamento de Aterosclerose da Sociedade Brasileira de Cardiologia. Arq Bras Cardiol. 2007;88:19.

15. Jelliffe DB. Evaluación del estado nutrición de la comunidad. Ginebra: Organización Mundial de La Salud; 1968.

16. World Health Organization. Obesity: preventing and managing the global epidemic. Report of a WHO Consultation. Geneva: World Health Organization; 2000

17. Richelsen B, Pedersen SB. Associations between different anthropometric measurements of fatness and metabolic risk parameters in non-obese, healthy, middle-aged men. Int J Obes Relat Metab Disord. 1995;19(3):169-74.

18. Kahn HS, Simoes EJ, Koponen M, Hanzlick R. The abdominal diameter index and sudden coronary death in men. Am J Cardiol. 1996;78(8):961-4.

19. Sampaio LR, Simoes EJ, Assis AM, Ramos LR. Validity and reliability of the sagittal abdominal diameter as a predictor of visceral abdominal fat. Arq Bras Endocrinol Metabol. 2007;51(6):980-6.

20. Lukaski HC, Bolonchuk WW, Hall CB, Siders WA. Validation of tetrapolar bioelectrical impedance method to assess human body composition. J Appl Physiol. 1986;60(4):1327-32.

21. Deurenberg P, Weststrate JA, Hautvast JG, van der Kooy K. Is the bioelectrical-impedance method valid? Am J Clin Nutr. 1991;53(1):179-81.

22. Lohman TG, Roche AF, Martorell R. Anthropometric standardization reference manual. Champaign: Human Kinetics Pub.; 1988.

23. Friedwald WT, Levy RI, Fredrickson DS. Estimation of the concentration of low-density lipoprotein cholesterol in plasma, without use of the preparative ultracentrifuge. Clin Chem. 1972;18(6):499-502.
24. Matthews DR, Hosker JP, Rudenski AS, Naylor BA, Treacher DF, Turner RC. Homeostasis model assessment: insulin resistance and beta-cell function from fasting plasma glucose and insulin concentrations in man. Diabetologia. 1985;28(7):412-9.

25. Hanley JA, McNeil BJ. A method of comparing the areas under receiver operating characteristic curves derived from the same cases. Radiology. 1983;148(3):839-43.

26. Barbosa PJB, Lessa Í, Almeida Filho N, Magalhães LBNC, Araújo J. Critério de obesidade central em população brasileira: impacto sobre a síndrome metabólica. Arq Bras Cardiol. 2006;87(4):407-14.

27. Weidner MD, Gavigan KE, Tyndall GL, Hickey MS, McCammon MR, Houmard JA. Which anthropometric indices of regional adiposity are related to the insulin resistance of aging? Int J Obes Relat Metab Disord. 1995;19(5):325-30.

28. Pouliot MC, Despres JP, Lemieux S, Moorjani S, Bouchard C, Tremblay A, et al. Waist circumference and abdominal sagittal diameter: best simple anthropometric indexes of abdominal visceral adipose tissue accumulation and related cardiovascular risk in men and women. Am J Cardiol. 1994;73(7):460-8.

29. Sjostrom L. A computer-tomography based multicompartment body composition technique and anthropometric predictions of lean body mass, total and subcutaneous adipose tissue. Int J Obes Relat Metab Disord. 1991;15 Suppl 2:19-30.

30. Salmenniemi U, Ruotsalainen E, Vanttinen M, Vauhkonen I, Pihiajamäki J, Kainulainen S, et al. High amount of visceral fat mass is associated with multiple metabolic changes in offspring of type 2 diabetic patients. Int J Obes Relat Metab Disord. 2005;29(12):1464-70.

31. Kahn HS. Alternative anthropometric measures of risk: possible improvements on the waist-hip ratio. In: Medeiros-Neto G, Halpern A, Bouchard $C$, editores. Progress in obesity research. $9^{\mathrm{a}}$ ed. London: John Libbey Eurotext Ltd.; 2003. p. 639-43.

32. Han TS, van Leer EM, Seidell JC, Lean ME. Waist circumference action levels in the identification of cardiovascular risk factors: prevalence study in a random sample. BMJ. 1995;311(7017):1401-5.

33. NCEP. Executive Summary of the Third Report of the National Cholesterol Education Program (NCEP) expert panel on detection, evaluation, and treatment of high blood cholesterol in adults (Adult Treatment Panel III). JAMA. 2001;285(19):2486-97.

34. Velásquez-Meléndez G, Gazzinelli A, Côrrea-Oliveira R, Pimenta AM, Kac G. Prevalence of metabolic syndrome in a rural area of Brazil. Sao Paulo Medical J. 2007;125(3):155-62.

35. Alberti KG, Zimmet P, Shaw J. Metabolic syndrome - a new world-wide definition. A Consensus Statement from the International Diabetes Federation. Diabet Med. 2006;23(5):469-80. 